\title{
Модели экономического влияния рекламы: три уровня эффектов 1
}

\section{Сергей Вартанов}

Московский государственный университет им. М.В. Ломоносова, Москва, Россия

\author{
Информация о статье \\ Поступила в редакичю: \\ 13.07.2020 \\ Принята \\ к опубликованию: \\ 13.11.2020 \\ УДК 51-77 \\ JEL C51, C52
}

\section{Ключевые слова:}

экономика рекламы, поведение потребителя, модели рекламной динамики, несовершенная конкуренция, медиарекламный рынок

\section{Keywords:}

advertising economics, consumer behavior, advertising dynamics models, imperfect competition, media advertising market

\begin{abstract}
Аннотация
Настоямая работа представляет собой первую, вводную часть ичикла статей, посвященных обзору существующцих моделей, методов и взглядов на эффекты воздействия экономического института рекламы на рынок на всех возможных уровнях. В первой части циикла приводится общий обзор наиболее значимых подходов к изучению рекламной деятельности фирм, влияния рекламы на потребителей и на характеристики экономического равновесия. Построена классификация экономических эффектов рекламы, учитывающая все существующие аспекты ее воздействия и кратко описаны подходы к анализу ее первичных, вторичных и третичных эффектов.
\end{abstract}

\section{Modeling Economic Impact of Advertising: Three Effect} Levels

\section{Sergey Vartanov}

\section{Abstract}

The present work is an introduction to a cycle of review articles dedicated to a comprehensive description and analysis of effects caused by advertising as an economic institution on the whole economy on different levels. A general review of most significant approaches to analyze the firms advertising activities is carried out as well as a diversified overview of methods and mathematical apparatus of modelling advertisement influence on consumers and on the characteristics of economic equilibrium. A classification of such influence effects is constructed, based on different characteristics of advertising, including its content, duration, intensity, as well as types of firms' ad strategies and the level of economy affected by ad influence. From the latter point of view the economic effects of advertising may be divided into three levels. Primary effects involve only the consumers and describe the shift in their demand inspired by advertisement. The emergence of primary effects gives a new tool to operate the demand for the producing firms. For the latter it means that they may develop an optimal strategy, different from the one that fits the "non-ad" case, and this difference

\footnotetext{
1 Работа выполнена за счет гранта Российского научного фонда (проект № 20-68-47030) DOI: https://dx.doi.org/10.24866/2311-2271/2020-3/45-66
} 
forms the essence of the secondary tier of the economic effects of the advertising (secondary effects). Finally, the tertiary effects of advertising deal with the shift in general market characteristics and structure induced by the existence of advertising as an institution such as social welfare change or emergence of new equilibriums or advertising markets as well as advertising-driven intersectoral linkages between production and media industries.

\section{Введение. Реклама как феномен неклассической экономики}

По своей природе реклама является неотъемлемой частью любой современной экономики. Рекламный контент проникает в жизнь потребителей со всех сторон - из телевизора, радио, прессы, из Интернета, с наружных носителей вроде рекламных щитов.

Реклама является одной из наиболее привычных форм коммуникации, которую можно использовать, чтобы сподвигнуть потребителей на те или иные действия, связанные с товаром. Чтобы повлиять на поведение потребителя, фирма может потратить средства на распространение рекламных сообщений через традиционные медиа - газеты, журналы, ТВ, радио, наружную рекламу, или через «новые» медиа - интернет, СМС-рассылку и т.д. В качестве основного инструмента убеждения покупателей реклама имеет две основных функции (Comanor, Wilson, 1974). Первая из них - быть «каналом» доставки до потребителя полезной информации, которая поможет ему принять рациональное решение, снижая информационную дифференциацию продукта. Вторая функция связана с «дифференцирующей» ролью рекламы, благодаря которой происходит в некотором смысле «обратный» процесс реклама убеждает потребителя с помощью нематериальных идей или психологических приемов в превосходстве соответствующего продукта, тем самым создавая продуктовую дифференциацию.

Существует большое количество работ, связанных с анализом рекламы и ее влияния на рыночные процессы. При этом подходы к рекламе в публикациях весьма различны и зависят, в первую очередь, от того, с позиций какого научного направления автор рассматривает феномен рекламы. Один из возможных вариантов «линии раздела» пролегает между работами более «гуманитарной» направленности (психология, социальная философия) и более «математизированными» работами (в первую очередь, это математическое моделирование экономик с рекламой). Однако при таком механистическом разделении теряется тот факт, что работы второй группы часто основаны на идеях и концепциях из первой группы. Поэтому несмотря на все многообразие математических моделей и применяемого в них теоретического аппарата (различные разделы теории игр, теория оптимизации, эконометрика), в основе классификации работ по математической экономике, связанных с рекламой должны лежать качественные особенности механизма воздействия рекламы на рынок и его участников.

Целью настоящей работы является обзор наиболее значимых работ по анализу рекламы, использующих весь доступный современной науке инструментарий - от исследования психологии принятия решений до стохастических дифференциальных уравнений динамики рынка. Несмотря на то, что существует ряд обзорных работ, связанных с экономикой рекламы, они, как правило, ограничиваются конкретным видом обозреваемых моделей, методо- 
логией анализа или временным промежутком. Например, обзор Huang, Leng, Liang (2012) концентрируется на описании работ по дифференциальным моделям рекламной конкуренции между производителями, имеющими возможность рекламировать свой товар, в то время как Назайкин (2014) предлагает обзор коммуникационных моделей рекламы, в основе которых лежит психологический подход к анализу принятия решений потребителями. Кроме того, существует ряд обзорных работ, имеющих еще более узкую направленность например, обзор Jørgensen, Zaccour (2014) описывает только работы, в которых исследуются теоретико-игровые модели рекламной кооперации в производственных цепочках. Таким образом, задача, ставившаяся при написании настоящей работы, является более широкой: автор постарается построить единую классификацию всех существующих на текущий момент методологий и подходов к анализу влияния рекламы на рынок на всех возможных уровнях и изложить основные результаты, полученные при их применении. Данная задача является невыполнимой в рамках одной статьи в силу широты и фундаментальности ее постановки, поэтому настоящая работа представляет собой цикл из пяти последовательных статей. Каждая из статей посвящена отдельному уровню экономических эффектов, оказываемых институтом рекламы на рынок в целом и его отдельных участников.

Влияние рекламы на рынок связано напрямую с потребителями и особенностями их индивидуального выбора под ее воздействием. В то же время, на поведение производителей реклама также оказывает заметное влияние, однако совершенно иной природы - для них она является одной из компонент их рыночной стратегии. Исходя из различного места рекламы в экономической деятельности производителей и потребителей, можно разделить все публикации по интересующей нас тематике на два крупных класса: работы, посвященные поведению потребителей в условиях рекламного воздействия, и работы, посвященные анализу поведения производителей (сюда также входят и статьи, связанные с медиапланированием). Необходимо отметить, что в работах второй группы поведение потребителей под влиянием рекламы также учитывается, однако основной акцент исследований делается именно на особенностях рекламного поведения фирм. Характерной особенностью этих исследований является то, что в них субъектами, принимающими решения, являются только фирмы. Конкретные особенности моделирования поведения потребителей варьируются от работы к работе - это может быть как единственный репрезентативный потребитель, так и несколько потребителей, существенно отличающихся своими характеристиками. Кроме того, очень часто вопрос внутренней мотивации и индивидуального выбора потребителей вообще «выносится за скобки», а их поведение описывается просто функцией спроса или функцией продаж, которая зависит помимо прочих рыночных характеристик товара (объема, цены, и т.д.) от рекламных затрат производителя.

В традиционной литературе существует три взгляда (если точнее, два «с половиной») на то, как реклама воздействует на потребителя, основной «водораздел» между которыми связан с ответом на вопрос «Почему потребители реагируют на рекламу?». Основная часть экономистов разделилась на три группы в соответствии с тем, как, по их мнению, необходимо ответить на данный вопрос, при этом каждое из развиваемых направлений в экономике рекла- 
мы обладает собственными дескриптивными и нормативными теориями. Первый взгляд на рекламу - информативный, он стал развиваться после 1960 года под влиянием трудов Чикагской школы. В рамках «информативного» подхода предполагается, что большая часть реальных рынков характеризуется неполнотой информации о товарах у потребителей, так как поиск данных о существовании отдельных товаров и их характеристик, как правило, связан с существенными затратами. Подобное информационное несовершенство приводит к рыночной неэффективности, однако не реклама является его причиной. Более того, в подобном контексте реклама становится в определенном смысле «лекарством» от этой неэффективности. Когда фирмы рекламируют свой товар, потребители могут получить информацию о них с минимальными затратами. Таким образом, спрос на товары рекламирующих фирм становится более эластичным, что повышает конкуренцию между ними. Кроме того, реклама может упростить вход фирм на рынок, так как позволяет сразу же проинформировать потребителей о существовании новой фирмы и нового товара на рынке.

Второе направление предполагает, что реклама убеждает потребителя это было доминирующее направление на протяжении первой половины двадцатого века. Убеждающий подход к экономике рекламы основан на предположении, что реклама изменяет вкусы потребителя и создает ложную дифференциацию продукта, превращая в товары-субституты, производимые под разными брендами, к разным товарам, и создавая лояльность к этим брендам. Следствием этого становится снижение эластичности спроса на товары фирм, действующих на рынке с рекламой, и, соответственно, рост цены на них. Кроме того, реклама товаров, производимых фирмами-старожилами рынка, создает дополнительные барьеры для входа на рынок новичков, которые становятся особенно тяжелыми для преодоления. Таким образом, «убеждающий» подход рассматривает рекламу как явление антиконкурентное, так как она не дает дополнительной «реальной» полезности для потребителей, взамен создавая искусственную дифференциацию продукта и приводя к появлению высоко концентрированных рынков, с повышенными ценами и прибылями производителей.

Еще один взгляд на рекламу заключается в том, что ей отводится дополняющая, комплементарная роль по отношению к самому товару. Согласно комплементарному подходу, реклама сама по себе не меняет предпочтений потребителя, как утверждается при убеждающем подходе, при этом она может не содержать вообще никакой информации о потребительских характеристиках товара (что автоматически «сводит на нет» ее информирующую роль). Вместо этого предполагается, что потребитель обладает устойчивыми предпочтениями на множестве характеристик товара, а реклама как бы «дополняет» это множество, увеличивая его размерность. Например, речь может идти о личном отношении потребителя к конкретным товарам или о престижности потребления товара: наряду с «традиционной» полезностью товара, которую потребитель получает от его потребления, он может испытывать дополнительное удовлетворение от осознания того, что потребляемый им товар принадлежит к «престижной» категории. В свою очередь, принадлежность товара к «престижной» группе задается как раз грамотно проводимой рекламной политикой. «Половинная» роль этого направления связана с тем, что при грубом обобщении комплементарную роль рекламы можно рассматривать как частный 
случай убеждающей - так как конечной целью ее воздействия, как и в «убеждающем» случае является воздействие на полезность потребителя (неважно, с помощью какого механизма и насколько глубоко это воздействие). С этой точки зрения убеждающий и комплементарный подходы к роли рекламы находятся «по одну сторону баррикад», противостоя информативному подходу.

Таким образом, в традиционной адвертологической литературе можно выделять несколько базовых подходов, каждый из которых можно воспринимать и как противоречащий остальным, и как дополняющий их. На самом деле, воздействие рекламы носит интегральный характер, в котором присутствуют и убеждающие элементы, и информирующие, и комплементарные. Более того, сам по себе вопрос анализа воздействия рекламы на принятие решений экономическими субъектами является частью более широкой задачи - изучения воздействия контекста на процесс принятия решений. Рассмотрение рекламного воздействия с подобной точки зрения исключает ее из числа объектов (нео)классической экономики, где основной действующий субъект - рациональный потребитель, действующий в условиях полной информации (в такой постановке рекламе не нашлось бы места). Реклама как один из способов манипулирования контекстом принятия экономических решений - вне зависимости от взглядов на механизм ее действия на потребителя - является в большей степени предметом институциональной (информативный подход рассматривает рекламу как средство сокращения транзакционных издержек, связанных с поиском информации) и поведенческой экономики (убеждающий и комплементарный подходы основаны, в первую очередь, на анализе воздействия рекламы на психоэмоциональное состояние потребителя, меняющем критерии принятия решения).

Классификация рекламных эффектов и описывающих их моделей: четыре «с половиной» уровня

Несмотря на различное толкование основных механизмов рекламного воздействия на принятие экономических решений отдельными индивидами все три подхода в экономической адвертологии сходятся в одном: конечная цель такого воздействия - потребитель, а источником воздействия являются фирмыпроизводители. Конечно, подобное деление экономических субъектов на производителей и потребителей весьма условно. Например, реклама банковских продуктов для бизнеса может быть направлена на компании-производители различных благ, однако с точки зрения рынка банковских кредитов они выступают как раз потребителями. С учетом этой оговорки можно рассматривать два уровня воздействия рекламы на рынок в целом. Первый уровень - непосредственное воздействие рекламы на потребителя, в результате которого он меняет свое поведение. Первичные эффекты рекламного воздействия связаны непосредственно с потребителями - как они изменяют свое поведение в условиях внешнего рекламного воздействия? В широком смысле под рекламой можно понимать манипуляцию контекстом принятия решения, не связанным с экономикой - то есть не цена, не характеристики товара и не рыночные институты.

Вторичные эффекты касаются изменения поведения производителей, связанное с появлением в их руках рекламы как нового инструмента воздействия на рынок - помимо классического управления ценой и характеристи- 
ками производимого товара. Ко вторичным эффектам относятся все «глобальные» эффекты от рекламного воздействия - изменение цен, объемов продаж, структуры рыночного равновесия и рынка в целом.

Другое направление классификации эффектов рекламного воздействия, не противоречащее, но дополняющее деление на первичное и вторичное воздействия, связано с временными рамками, в течение которых осуществляется реклама. Краткосрочные (мгновенные) эффекты связаны с рекламным воздействием небольшой продолжительности, а долгосрочные эффекты отражают воздействие длительных и планомерных рекламных кампаний. В целом подобное разделение эффектов рекламы на мгновенные и долгосрочные тесно сопряжено с предположением о наличии у потребителей долгосрочной памяти о потребленных товаров. Дело в том, что в случае долгосрочного рекламного воздействия информирующее воздействие рекламы практически уходит «на второй план», так как на рынке появляются так называемые «опытные» потребители - те, которые знают о точных характеристиках товара на основании личного опыта их потребления. На таких потребителей воздействие рекламы может носить только убеждающий или комплементарный характер. Помимо особенностей, связанных с опытом потребления рекламируемых товаров, который «вредит» информирующему воздействию, есть эффекты, снижающие и убеждающее/комплементарное воздействие рекламы при наличии у потребителя долгосрочной памяти - он может помнить не только о характеристиках товара, но и совершенном на него в прошлом рекламном воздействии. В качестве примера подобного эффекта можно привести раздражение от рекламы (annoying effects), возникающее при излишне навязчивой, длительной и однообразной рекламе. Таким образом, наличие у потребителей памяти и ее продолжительности также является одним их «краеугольных камней» в классификации эффектов рекламного воздействия и описывающих их моделей.

Кроме того, отдельный уровень классификации рекламных моделей связан с особенностями самой рекламы и того, как она доставляется до потребителя. Особенности человеческого восприятия обуславливают различия в воздействии на потребителя рекламной информации, «ориентированной» на различные органы его чувств. Так как и убеждающее, и комплементарное действие рекламы основаны в первую очередь на воздействии на эмоции потребителя, очевидно, что рекламные тексты, изображения и звуковые ролики, а также видеоклипы приводят, вообще говоря, к различным эффектам. Информирующее воздействие рекламы также может иметь различную эффективность в зависимости от того, на какие органы чувств ориентировано рекламное сообщение (например, для некоторых потребителей инфографика предпочтительнее «голого» текста). Таким образом, воздействие текстовой, аудиальной, визуальной рекламы и их комбинаций носит, вообще говоря, различный характер и описывается различными моделями.

В ряде ситуаций особенности рекламы могут явно определять основной механизм ее воздействия - например, объявления в газете или на онлайндосках оказывают преимущественно информирующее действие, а высокобюджетные рекламные ролики для телевидения направлены на увеличение престижности потребления рекламируемого товара, что является частным случаем комплементарного воздействия. Кроме того, сами каналы, по кото- 
рым реклама доставляется до потребителя имеют значение с точки зрения оказываемых рекламой эффектов. Например, в работах, посвященных интернет-рекламе и телерекламе используются совершенно различные модели их воздействия, кроме того, существует ряд работ (например, Prasad and Sethi, 2009), в которых распространение рекламы возможно сразу по нескольким каналам (ТВ, печатные СМИ, рекламные брошюры, наружная реклама - то, что традиционно относится к ATL-рекламе ${ }^{2}$ ). Помимо различных каналов доставки рекламной информации, существует еще один способ рекламной деятельности, который условно можно считать отдельным аналогом канала доставки. Речь идет о продвижении товара «на местах» - то есть в магазинах, салонах продаж и т.п. Сюда же можно отнести всю так называемую BTLрекламу (below the line) ${ }^{3}$. Огромное количество литературы посвящено анализу совместного воздействия «традиционной» рекламы и продвижения товара в местах продаж. Особенно плодотворным в существующей литературе оказался подобный анализ для вторичного воздействия рекламы на рынках с продуктовыми цепочками типа «производитель-ритейлер».

Таким образом, можно разделить все обсуждающиеся в литературе эффекты рекламного воздействия на несколько крупных классов, в зависимости от того, какими свойствами они обладают. Всего возможны четыре уровня классификации, практически независимые друг от друга.

1. Уровень воздействия рекламы:

а. первичные эффекты, связанные с изменением потребительского поведения по сравнению с неоклассической задачей потребителя;

b. вторичные эффекты, связанные с изменением рыночного поведения производителей, вызванным изменением спроса в результате первичных эффектов;

с. третичные эффекты, связанные с изменением свойств рыночного равновесия и возникновением новых, не существующих на рынках без рекламы, рыночных структур.

2. Преимущеественный механизм воздействия на потребителей:

а. информативный;

b. неинформативный:

i. убеждающий;

ii. комплементарный.

3. Особенности доставки рекламы до потребителя:

а. канал доставки информации:

i. телевизионная, печатная реклама и реклама на радио (традиционные СМИ);

ii. интернет-реклама, социальные сети и т.д. (новые СМИ);

iii. продвижение товаров в местах продажи (промоушн).

b. вид рекламной информации

i. текстовая реклама (объявления, сообщения);

${ }^{2} \mathrm{ATL}$ (от англ. above-the-line) - комплекс маркетинговых коммуникаций, включающий традиционные (классические) виды рекламы. В ATL включается реклама в традиционных СМИ - пресса, радио, телевидение, реклама OOH (англ. Out Of Home) - наружная и внутренняя, а также полиграфическая реклама.

${ }^{3}$ BTL-реклама (точнее, BTL-маркетинг) - это комплекс маркетинговых коммуникаций, который отличается от прямой рекламы ATL способом воздействия на целевую аудиторию. Как правило, инструменты BTL позволяют контактировать с участниками промо-акций лично, непосредственно в точках продаж. 
ii. аудиовизуальная реклама (изображения, видео, звуковые ролики).

4. Временной горизонт эффектов:

a. мгновенные эффекты (например, эмоциональная спонтанная покупка ненужного товара);

b. долгосрочные эффекты (формирование бренда и связанного с ним образа, наличие у потребителя знаний о свойствах товара).

Приведенная схема классификации носит не иерархический характер и, конечно, является довольно грубой, однако она позволяет описать практически все исследованные эффекты рекламного воздействия. Более того, опираясь на предлагаемый способ описания рекламных эффектов, можно классифицировать и изучаемые в литературе последнего времени модели рекламы. При этом возникает дополнительный уровень классификации (номер «четыре с половиной»), связанный уже в большей степени с методикой и методологией анализа рекламного воздействия, чем с особенностями самого этого воздействия. Этот уровень классификации является скорее техническим, нежели сущностным, однако автор считает необходимым по возможности учитывать и его. Речь идет о классификации моделей рекламного воздействия по методам их построения и анализа, и на этом уровне классификация моделей уже существенно зависит от того, какому из типов более высокого уровня они принадлежат. На данном уровне классификации, например, может проводиться разделение на качественные и количественные модели. Так, например, коммуникативные модели рекламы представляют собой пример качественных моделей первичного воздействия рекламы. В свою очередь, они могут подразделяться на другие подклассы в зависимости от особенностей механизма воздействия рекламы на потребителя и особенностей памяти потребителя. Качественный характер таких моделей обуславливает их в большей степени философский, чем экономический характер. С другой стороны, существует и большое количество работ, посвященных именно экономическому анализу первичного воздействия рекламы и опирающиеся на математические модели принятия решения (аналитические или эконометрические).

Что касается моделей вторичного воздействия рекламы, то здесь уровень классификации «номер четыре с половиной» связан с качественными различиями рынков, для которых производится анализ рекламного воздействия. На этом уровне проявляются различия между моделями рекламы на монопольных и олигополистических рынках, а также моделями рынков с фирмами, объединенными в производственные цепочки.

\section{Первичные эффекты воздействия рекламы: общие рамки и методы исследования}

Как уже говорилось ранее, первичные эффекты рекламного воздействия характеризуют то, как меняется выбор потребителей в условиях внешнего рекламного воздействия. К работам, изучающим подобные эффекты, можно отнести широкий спектр литературы, основанный на весьма разнообразных как по своей сущности, так и по методологии моделях. Более того, в рамках исследования моделей первичного воздействия рекламы само понятие рекламы трактуется максимально широко: не только как доставляемые потребителю по различным каналам сообщения о товаре, но и как манипуляцию контекстом 
принятия решения. Благодаря такой широте толкования, спектр исследуемых вопросов и методов анализа первичного воздействия рекламы также крайне разнообразен. К моделям первичного воздействия рекламы можно отнести как коммуникативные модели рекламы, описывающие на качественном уровне процесс воздействия рекламы на потребителя и индуцированные им изменения в его поведении, так и микроэкономические модели потребительского выбора, модифицированные с учетом рекламного воздействия.

Хронологически самой первой и уже ставшей классической работой, где обсуждалось влияние рекламы на потребителя, является книга Чемберлена (Chamberlin, 1933). Чемберлен считал, что реклама влияет на спрос благодаря двум аспектам:

1. Она доставляет до потребителей информацию о существовании товара, его производителя, а также о некоторых рыночных характеристиках (цена, качество);

2. Она изменяет вкусы и предпочтения потребителя.

Именно эти два названные Чемберленом фактора влияния рекламы в дальнейшем нашли развитие в двух подходах к роли рекламы - информирующем и убеждающем. Если в случае с информирующим действием рекламы ее главным эффектом является снижение информационной асимметрии среди участников рынка, то для убеждающего действия эффекты более разнообразны. В этом случае могут измениться не только предпочтения потребителя, но и сама схема принятия им решения - вплоть до того, что для описания его поведения оказывается неприменимым классический подход, основанный на предположении о рациональности потребителя и наличии у него устойчивых предпочтений на множестве рыночных альтернатив (товаров). Иногда рекламное воздействие носит «отрицательный» характер - вместо того, чтобы сообщать о характеристиках конкретного товара (информирующее воздействие) или склонять выбор потребителя в сторону данного товара (убеждающее воздействие), рекламные сообщения бывают направлены на «отвращение» потребителей от товаров-конкурентов. Такое воздействие также может быть информативным (например, информировать о негативных последствиях потребления товара), убеждающим (в основном, запугивание и негативное воздействие на эмоции) или комплементарным (формирование негативного, немодного образа брендаконкурента). Однако, вне зависимости от того, стоит ли задача склонить потребителя к выбору товара, или, напротив, отвратить его от него, главная цель рекламы заключается в том, чтобы подтолкнуть потребителя к тому решению, которое необходимо рекламодателю.

В процессе рекламной коммуникации поведение потребителя представляет собой упорядоченную последовательность эффектов - реакций потребителя на рекламу. В общем случае такая последовательность имеет вид: реклама - промежуточные эффекты - покупка. Следующая в иерархии потребительская реакция всегда наступает только после реализации предыдущей. Традиционно выделяется три основных типа иерархии принятия решений потребителем в условиях рекламной коммуникации в зависимости от типа рекламируемого товара и особенностей подачи рекламных сообщений: иерархия с высокой вовлеченностью, иерархия с низкой вовлеченностью, а также иерархический процесс рационализации (Ray, 1973). Иерархия с высокой вовлеченностью ба- 
зируется на цепочке действий «узнал - почувствовал - сделал» (три уровня расположены в иерархической последовательности «когнитивный - аффективный - конативный»). Согласно обобщающему иерархические модели с низкой вовлеченностью подходу, реклама обращена преимущественно к существующим пользователям марки и нацелена на усиление их привязанности к ней. Потребители в таких моделях обладают динамически изменяющимися предпочтениями, которые в каждый момент времени позволяют ранжировать все существующие на рынке товары с точки зрения их привлекательности для потребителя. При этом главным долгосрочным эффектом рекламного воздействия является сохранение и, по возможности, улучшение места рекламируемого товара в таком «рейтинге». Третий класс иерархических моделей рекламной коммуникации - модели рационализации - предполагает, что реклама воздействует на потребителя неявно, оставляя его в полной убежденности, что он совершает свой выбор только на основе максимизации своей полезности.

Все иерархические модели рекламной коммуникации базируются на нескольких общих принципах. Во-первых, все они в качестве основного механизма воздействия рекламы на потребителя рассматривают убеждение. Вовторых, процесс принятия решения потребителем представляет собой последовательность изменений его состояния под воздействием рекламы. При этом потребитель может не запоминать совершенные ранее действия и полученную рекламную информацию (тогда модель описывает процесс однократного контакта с рекламой и последующей покупки товара), а может и обладать памятью, и помнить всю рекламу, которую видел ранее (в этом случае иерархические модели итеративно описывают процесс многократного потребления рекламы и последующей покупки товара).

Помимо классических каналов доставки рекламы (различные медиа: телевидение, радио, интернет, и т.д.), в качестве «аналога» канала доставки можно рассматривать маркетинговые мероприятия, проводимые уже в точках реализации товара - то есть в магазинах, салонах продаж и т.п. Для описания особенностей принятия потребителями решений, связанных с рекламным воздействием прямо в момент покупки товара, в последнее время используются преимущественно методы поведенческой экономики. Основное отличие в подходе к моделированию такого воздействия от иерархических моделей заключается как раз в одновременности принятия решения о покупке и рекламного воздействия. Эта одновременность приводит к тому, что горизонт принятия решения потребителем становится не просто краткосрочным, но практически мгновенным. На таком временном интервале невозможно построить рекламную коммуникацию традиционными способами - например, через СМИ. Таким образом, для ряда задач, связанных с продвижением товара в местах реализации и/или с принятием решения о количестве приобретаемого потребителем товара (т.е. товар уже выбран из ряда аналогов), традиционные модели эффектов рекламной коммуникации могут оказаться неприменимы. В этом случае используются модели, основанные на постулатах поведенческой экономики. Еще одно направление, по которому различаются эффекты рекламного воздействия, связано с каналом восприятия информации потребителем, по которому он получает рекламу. Здесь речь идет, в первую очередь, о различии в воздействии на потребителя аудиовизуальных образов 
и информации, получаемой им вербально (в первую очередь, в виде текста). Существует еще одно «измерение» - внешний вид самого товара - позиционирование, в котором может оказать влияние на потребительское поведение.

\section{Вторичные эффекты воздействия рекламы: изменение поведения фирм}

При обсуждении первичных эффектов рекламного воздействия «фокус» исследования был зафиксирован на изменении поведения потребителя. Однако при описании и анализе всех воздействующих на потребителя рекламных эффектов, как правило, «выводился за скобки» тот факт, что интенсивность и способ проведения рекламной кампании, вообще говоря, есть результат стратегического поведения фирмы, выпускающей (и/или продающей) соответствующий товар. Так как реклама оказывает существенное влияние на поведение потребителя, почти любому производителю приходится осуществлять связанные с ней затраты (например, на рекламные кампании в СМИ), чтобы «продвигать» свой товар. Однако эти затраты, как и другие, сдерживаются бюджетными ограничениями. Таким образом, для производителя оптимальные затраты на рекламу должны быть решениями оптимизационной задачи максимизации общей прибыли за счет повышения объема продаж. Так как в реальности воздействие рекламы обычно меняется с течением времени, возникает важный вопрос для исследования: как фирма должна распределять свои (ограниченные, вообще говоря) затраты во времени, чтобы увеличить продажи или максимизировать итоговую прибыль? Чтобы решить подобную задачу, необходимо, в первую очередь, использовать адекватную модель воздействия рекламы на потребителей, чтобы описать изменение уровня продаж по сравнению с «безрекламным» случаем.

Многие исследователи предпочитают фокусироваться на математическом анализе моделей рынков с рекламой, описывая их формально или вообще ссылаясь на формулировку одной из базовых моделей. На самом деле, существует очень ограниченное количество таких базовых моделей, и практически любая изучаемая в литературе модель рынка по факту является частным случаем или модификацией одной из них. Каждая из базовых моделей основана на определенных предпосылках относительно поведения потребителей под воздействием рекламы - то есть на том, какой из первичных эффектов рекламного воздействия предполагается основным. Так, модели Видаля-Вольфа можно интерпретировать как основанные преимущественно на краткосрочном убеждающем воздействии рекламы - так как воздействие рекламы в нем предполагается моментальным и хранящимся в памяти потребителя не слишком долгое время. Это соответствует обсуждавшимся в предыдущей главе краткосрочным эффектам убеждающей рекламы как продуктам мгновенного эмоционального решения. А вот модели Нерлова-Эрроу характеризуются в большей степени долгосрочным воздействием на потребителя, в основе которого лежит эффект престижа. Концепция гудвилла, лежащая в основе всех таких моделей, показывающая накопленное за все время потребления товара и контактов с рекламой желание потребителя купить (willingness-to-pay), довольно точно соотносится с идеей о престиже потребления товара, как новой потребительской характеристики. Более того, сам по себе запас гудвилла товара можно рассматривать как меру его престижности - хотя бы в первом приближении. 
Как и в случае первичных эффектов, принципиальную важность для математических моделей вторичных эффектов рекламы несут временные рамки их действия. Способность потребителей запоминать информацию о товаре становится одной из основ классификации таких моделей. Дело в том, что рынки с потребителями, не способными запоминать прошлые рекламные сообщения или свойства товара, потребленного ранее, могут быть описаны моделями рыночной конкуренции, сводящимися, как правило, к играм в нормальной форме с фирмами-производителями в качестве игроков. В то же время, для описания рынков, где потребители способны накапливать опыт потребления - как товаров, так и рекламы - потребуются уже модели, «протяженные во времени». В основном в качестве математического аппарата анализа таких моделей используются дифференциальные игры, где в качестве решения выступают различные варианты динамического равновесия - как правило, это равновесие Нэша с обратной связью или без нее.

В рамках первого направления предполагается, что все потребители не имеют памяти и при покупке ориентируются только на рекламу, сделанную в текущем временном интервале. Такое направление можно условно считать «статическим», потому что в таких моделях фирмы-игроки принимают решения, затрагивающие только один период. Так как решения, принятые фирмами в прошлом, не играют для потребителей роли, основные параметры моделей - спрос, прибыль фирм, структура рынка - фиксированы и не зависят от времени. В работах второго направления предполагается, что потребители способны накапливать опыт потребления - как товара, так и рекламной информации. Модели, изучаемые в работах этого направления, основаны уже на динамических моделях теории оптимизации (для монопольных рынков) и теории игр (для рынков с количеством фирм более двух). В таких моделях рекламные стратегии, применявшиеся фирмами в более ранних периодах, оказывают влияние на потребительское поведение в последующем - так как потребители помнят, вообще говоря, не только всю рекламу, с которой контактировали, но и всю историю рыночного поведения фирм. При этом особенности накопления потребителями опыта и его влияния на будущий спрос существенно отличаются для различных постановок задачи.

Для каждого класса моделей можно описать базовые принципы и рекламные эффекты, на которых базируется их математическая формализация. Модели рынков с рекламой основываются на классических микроэкономических моделях рынка. Рекламное воздействие дает производителям возможность воздействовать на спрос потребителей, безотносительно того, какой характер оно носит. Так, информативное воздействие изменяет (расширяет) у потребителя множество допустимых альтернатив, что может привести к изменению и бюджетного множества и, как следствие, изменению решения задачи потребителя и, соответственно, функции его спроса (информирующий эффект). Неинформативное рекламное воздействие изменяет предпочтения потребителя (например, эффект престижа), тем или иным способом повышая для него привлекательность рекламируемого товара, что также ведет к изменению вида задачи потребителя и, в конечном счете, функции спроса. Таким образом, вне зависимости от особенностей рекламного воздействия и от того, какой первичный эффект предполагается основным, спрос каждого потреби- 
теля является функцией от рекламных стратегий фирм. Кроме того, предполагается, что каждый потребитель является ценополучателем, а поведение потребителей в целом описывается с помощью совокупной функции спроса.

В основе всех математических моделей рынков с рекламой лежат базовые модели рынков, характерные для микроэкономики (в основном, модели монополии, олигополии). Базовым при их построении является предположение о дифференциации товара - потребители могут различать товар, произведенный различными фирмами. При этом товары различных производителей, как правило, предполагаются субститутами, а поведение всех потребителей на рынке агрегируется с помощью введения репрезентативного потребителя, на поведение которого и воздействует реклама. В общем случае спрос такого репрезентативного потребителя на товар, производимый фирмой $i$, имеет вид: $D_{i}=D_{i}\left(\mathbf{p}_{i}, \mathbf{p}_{-i} ; \mathbf{a}_{i}, \mathbf{a}_{-i} ; \mathbf{z}\right)$. Здесь $p_{i}-$ цена этого товара, вектор $\mathbf{a}_{i}$ характеризует стратегию его рекламы, $\mathbf{p}_{-i}$ - вектор цен на другие товары (продукцию конкурентов), а вектор $\mathbf{a}_{-i}$ составлен из параметров рекламы этих товаров; $\mathbf{z}$ - вектор прочих параметров модели. В некоторых моделях вместо функции спроса используется функция объема продаж или рыночная доля фирмы, при этом набор аргументов у этой функции тот же.

Поведение фирм на рынке описывается, в первую очередь, рекламной стратегией. В простейших моделях в качестве таковой обычно рассматривается уровень затрат на рекламу или интенсивность рекламного воздействия, в случае динамического рынка речь идет о зависящей от времени функции управления, описывающий временной план подобных затрат (т.е. $\mathbf{a}_{i}=\mathbf{a}_{i}(t)$ выступает в роли переменной управления, а объем продаж/спрос - в роли фазовой переменной). Дальнейшее усложнение моделей может происходить по двум направлениям, соответствующим расширению возможностей фирм. Вопервых, ряд моделей предполагает, что рекламная стратегия фирм представляет собой не просто совокупный объем затрат на рекламу, но вектор, характеризующий затраты на рекламу разных видов и типов. Во-вторых, стратегия фирмы помимо рекламы может включать в себя классические рыночные компоненты - как правило, это цена. В моделях рынков с рекламой традиционно предполагается, что помимо рекламной, фирмы сталкиваются с ценовой конкуренцией (а не с объемной). В частности, если зафиксировать рекламные стратегии всех фирм (то есть исключить возможность управлять рекламой), то, согласно стандартному подходу к анализу рынков с рекламой, базовой моделью для описания рынка будет модель ценовой конкуренции с дифференциацией товара (вертикальной, горизонтальной либо с комбинацией этих двух вариантов). Если на рынке присутствует всего лишь одна фирма, то базовой моделью является классическая модель монополии, если фирм несколько - то речь идет о модели олигополии в условиях ценовой конкуренции с дифференциацией товара. В отличие от широко изучаемой в литературе модели монополистической конкуренции, где товар так же дифференцирован, в качестве рыночной стратегии предприятий рассматривается цена, а не объем производимого товара.

Общим подходом при построении моделей рынков с рекламой является то, как формируется выигрыш фирм. В его качестве обычно берется прибыль 
- то есть цена товара, умноженная на объем проданного товара, минус издержки на производство и рекламные затраты. В зависимости от того, предполагается ли наличие памяти у потребителей и является ли рынок статическим или динамическим, в качестве целевой функции фирм выступает либо сама по себе, либо же ее интеграл по всему интервалу планирования. В общем виде для статического рынка она будет иметь вид:

$$
\pi_{i}(\mathbf{p} ; \mathbf{a} ; \mathbf{z})=p_{i} \cdot D_{i}(\mathbf{p} ; \mathbf{a} ; \mathbf{z})-c_{i}\left(D_{i}(\mathbf{p} ; \mathbf{a} ; \mathbf{z})\right)-\varphi_{i}\left(\mathbf{a}_{i}\right)
$$

где $\mathbf{p}=\left(p_{i}, \mathbf{p}_{-i}\right)-$ вектор цен на все товары, $\mathbf{a}=\left(\mathbf{a}_{i}, \mathbf{a}_{-i}\right)-$ совокупность рекламных стратегий всех фирм, $c_{i}(\cdot)$ - функция полных производственных затрат фирмы $i, \varphi_{i}(\cdot)$ - функция рекламных затрат в зависимости от выбранной стратегии $\mathbf{a}_{i}$ (так, если в качестве стратегии рекламы единственного товара выступает объем расходов на его рекламу, то $\left.\varphi_{i}(a) \equiv a\right)$.

В случае динамического рынка каждая фирма максимизирует интегральный функционал вида:

$$
J_{i}(\mathbf{p}(\cdot), \mathbf{a}(\cdot))=\int_{0}^{T}\left\{p_{i}(t) \cdot q_{i}(t)-c_{i}\left(q_{i}(t)\right)-\varphi_{i}\left(\mathbf{a}_{i}(t)\right)\right\} e^{-\delta t} d t
$$

где $p_{i}(t)$ - мгновенное значение цены на товар фирмы $i, \mathbf{a}_{i}(t)$ - ее рекламная стратегия в момент времени $t$, а $q_{i}(t)$ - мгновенное значение продаж товара фирмы $i$, динамика которого описывается дифференциальным уравнением вида (по сути, это ограничения для возникающей задачи оптимального управления):

$$
\begin{gathered}
\dot{q}_{i}(t)=d_{i}(\mathbf{p}(t), \mathbf{a}(t), \mathbf{z}(t)) \\
q_{i}(0)=q_{0}
\end{gathered}
$$

Необходимо отметить, что приведенный вид является максимально общим, а функция $d_{i}(\cdot)$ имеет смысл мгновенного приращения спроса (спроса в мгновенном периоде) при текущих параметрах рынка. Так, дифференциальное уравнение, задающее динамику продаж товара, может быть как детерминированным (тогда оно имеет приведенный выше вид), так и стохастическим, тогда его можно записать в виде:

$$
d q_{i}(t)=d_{i}(\mathbf{p}(t), \mathbf{a}(t), \mathbf{z}(t)) d t+\sigma d \omega(t),
$$

где $\omega(t)$ - случайный процесс с известными характеристиками.

Таким образом, в случае монопольного рынка задача фирмы представляет собой задачу оптимизации (для статического рынка) либо задачу оптимального управления (для динамического рынка). В случае олигополистического рынка базовая модель приобретает теоретико-игровой характер; в статическом случае базовая постановка задачи заключается в поиске равновесия Нэша, в динамическом задача относится к классу дифференциальных игр, и в качестве ее решения выступают равновесие Нэша в виде как замкнутого управления (closed-loop Nash equilibrium, стратегии не предусматривают коррекцию в зависимости от текущего значения параметров рынка), так и управления с обратной связью (open-loop Nash equilibrium, стратегии являются функциями не только от времени, но и от текущего значения параметров рынка). 
Любой вторичный эффект определяется, с одной стороны, первичными эффектами, действующими на потребителя, с другой стороны, моделью рыночного взаимодействия на конкретном исследуемом рынке. Это позволяет построить классификацию вторичных эффектов на основе классификации первичных и классификации моделей рынков. Все рассмотренные до настоящего времени математические модели рынков могут быть преобразованы к одному из описанных базовых классов. Различный вид конкретных моделей определяется уже особенностями рекламного воздействия, то есть ответом на вопросы: какой из первичных эффектов носит превалирующий характер, какой горизонт планирования рекламной кампании у фирм, каковы свойства памяти потребителей, какие каналы доставки информации доступны фирмам и так далее. Это связано с тем, что математическая модель рыночного взаимодействия, вообще говоря, определяется структурой рынка и особенностями рекламного воздействия на потребителя. Теми же самыми факторами определяются и вторичные эффекты рекламного воздействия - следовательно, можно классифицировать вторичные эффекты рекламного воздействия, как и математические модели рынка, не по формальным «математическим» критериям моделей, описывающих рынки, где возникают эти эффекты, а по «сущностной» классификации, в основе которой лежат классификация первичных эффектов, приводившаяся в самом начале настоящей работы, и свойства структуры рынка.

В качестве верхнего уровня классификации вторичных эффектов рекламного предлагается рассматривать «временной» уровень, основанный на двух факторах: сроке действия первичных эффектов рекламного воздействия (кратко-/долгосрочные) и на горизонте планирования рекламной кампании фирм (также кратко-или долгосрочные). Скомбинировав эти факторы, получим следующую таблицу, описывающую три группы вторичных эффектов (табл. 1).

Таблица 1

Классификация эффектов рекламного воздействия в зависимости от комбинации сроков планирования и сроков действия первичных эффектов

\begin{tabular}{|c|c|c|}
\cline { 2 - 3 } \multicolumn{1}{c|}{} & $\begin{array}{c}\text { Краткосрочные первичные } \\
\text { эффекты }\end{array}$ & $\begin{array}{c}\text { Долгосрочные первичные } \\
\text { эффекты }\end{array}$ \\
\hline $\begin{array}{c}\text { Колкосрочное планирование } \\
\text { фирм }\end{array}$ & \multicolumn{2}{|c|}{ Статические (вневременные) эффекты } \\
\hline $\begin{array}{c}\text { дирм планирование } \\
\text { фирмес }\end{array}$ & Эффекты мгновенной реакции & Накопительные эффекты \\
\hline
\end{tabular}

Первый класс вторичных эффектов - статические (вневременные) эффекты. Они возникают на тех рынках, где фирмы планируют свою деятельность лишь на один период вперед. В этом случае на принятие фирмой решения не оказывают влияние долгосрочные первичные эффекты. Название «статические» этому типу эффектов дано из-за того, что задача, решаемая фирмой при принятии решения, является статической и не учитывает возможного влияния избранной стратегии - как рыночной, так и рекламной - на потребительское поведение в последующих периодах. С точки зрения математического аппарата, используемого для анализа моделей таких рынков, задача исследования вторичных эффектов рекламного воздействия относится к задачам сравнительной статики, возникающих при анализе однократного ры- 
ночного взаимодействия (как уже говорилось, они основаны либо на оптимизационных моделях, либо на теоретико-игровых). Примерами статических вторичных эффектов являются правило ценообразования ДорфманаШтайнера ${ }^{4}$ и эффект входного рекламного барьера ${ }^{5}$.

В том случае, если фирмы-участники рынка планируют свою стратегию на долгосрочную перспективу, то помимо краткосрочных первичных эффектов они уже должны учитывать и долгосрочные. В зависимости от того, какие первичные эффекты доминируют на данном рынке, вторичные эффекты можно разделить на эффекты мгновенной реакции и на накопительные эффекты. Вторичные эффекты мгновенной реакции возникают на тех рынках, где поведение потребителей определяется в основном краткосрочными первичными эффектами - поведенческими, убеждающими и т.д. Одним из примеров вторичных эффектов мгновенной реакции является эффект пульсации рекламной кампании (фирмы чередуют периоды интенсивной рекламы с периодами «тишины»). Если основные первичные эффекты рекламы носят долгосрочный характер, то на рынке возникают вторичные эффекты накопительного характера. К ним относятся, например, гудвилл-эффекты и эффект управляемой рекламной диффузии. Особенности долгосрочного планирования фирмами своих стратегий обуславливают класс математических задач, к которым относится задача моделирования эффектов рекламного воздействия для таких рынков - это задачи динамики рынка, основанные на теориях оптимального управления и дифференциальных игр.

Большая часть существующих к настоящему моменту обзорных работ ограничивается описанием и классификацией именно математических моделей рекламного воздействия. Классификация таких моделей проводится, как правило, по формальным математическим свойствам, связанным с особенностями алгебраических или дифференциальных уравнений, лежащих в основе самих моделей. В настоящей работе же предлагается взглянуть на вторичные эффекты рекламы и математические модели рынков, где они возникают, с тех же позиций, что и на первичные, и разделить их по схожим уровням классификации - по особенностям памяти потребителей, по преимущественному механизму воздействия на потребителей и по особенностям доставки рекламы до потребителя. Кроме этого, в предлагаемую классификацию моделей рынков с рекламой добавляются еще уровни, связанные с особенностями производителей на этих рынках. Речь идет о разделении моделей на рынки с продажей товара потребителям непосредственно самими фирмами (классические модели рекламной монополии и олигополии) и на рынки с производственными цепочками, состоящими из производителей товара и специализированных ритейлеров. Однако особенности поведения ритейлеров как рекламных посредников и связанного с рекламой их взаимодействия с производителями позволяют отнести возникающие в этом случае эффекты к третичному уровню.

\footnotetext{
${ }^{4}$ Подробнее о модели Дорфмана-Штайнера (Dorfman, Steiner, 1954) рассказывается в последующих частях настоящего обзора; одноименное правило, упрощенно говоря, является «рекламным аналогом» правила обратной эластичности для монопольного ценообразования: оптимальные уровни цен и рекламных расходов должны уравнивать долю рекламных затрат и отношение рекламной и ценовой эластичностей спроса.

5 Этот эффект возникает, когда вход на рынок новых фирм осложняется за счёт высоких затрат на рекламу - как информативную (сообщить достаточному для рентабельности количеству потребителей о новом товаре слишком дорого), так и убеждающую (продукция имеющихся компаний не может быть равнозначно заменена товаром компании с новым, недостаточно раскрученным брендом).
} 


\section{Третичные эффекты и возникновение медиарекламного рынка}

Проанализированный в рамках настоящего обзора объем научной литературы характеризуется крайней широтой как социально-философских, так и методологических подходов к анализу рекламы и ее места в современной экономике. При этом воздействие ее на структуру и исход взаимодействия участников любого рынка, где существует возможность рекламы, столь значительно и многопланово, что нам пришлось построить многоуровневую классификацию рекламных эффектов. При этом самое значительное различие - дающее нам самый «верхний» уровень классификации - связано с тем, на рыночных агентов какого типа оказывается влияние рекламы. Первичные эффекты сказываются на потребителях и их предпочтениях, вторичные - уже на производителях, их стратегиях и, как следствие, на общих характеристиках структуры рынка. Однако, на самом деле, существует и более высокий уровень влияния рекламы на рынок. Он связан уже не с изменением в поведении существующих участников рынка под влиянием отдельных типов рекламного воздействия, а с ролью и метом рекламы как экономического института. Этот эффект заключается в возникновении рынка рекламы и, более широко, рынка медиа в целом. Этот рынок не является классическим товарным рынком, ибо на нем «продается» аудитория - то есть возможность организовать рекламное воздействие любого типа со стороны любого производителя на любого потребителя. При этом каналы доставки информации в такой постановке перестают быть «инструментом» рекламного воздействия и обретают субъектность, а «старые» участники рынка приобретают новые роли: потребители обретают статус аудитории, а производители - статус рекламодателей. В отличие от классических микроэкономических моделей, основанных на взаимодействии двух групп экономических агентов, каждая из которых имеет одну роль, здесь возникает некий «метарынок» с тремя сторонами, каждая из которых имеет несколько ролей.

Проводившийся в существующей литературе анализ, как правило, касался только одной из «сторон» рекламного воздействия - или потребителей и их индивидуальной реакции на рекламу, или изменения рыночной стратегии производителей с учетом такой потребительской реакции. Так, работы первого направления - «потребитель-ориентированного» - описывали особенности влияния рекламы на задачу потребителя и первичных эффектов ее воздействия. Работы второго направления - «производитель-ориентированного» - исследовали рынки товаров, на которых производители проводили рекламную и маркетинговую политику с целью воздействия на продажи, и были посвящены эффектам, названным нами вторичными. В этих работах потребители представлялись в виде своего рода «черного ящика», а рекламная стратегия производителей либо в явном виде задавалась их затратами на рекламу и маркетинг, либо сводились к ним.

Классические модели рекламной динамики - Видаля-Вольфа (Vidale, Wolfe, 1957), Нерлова-Эрроу (Nerlove, Arrow, 1962) - и их производные вообще не оставляют места для описания индивидуального поведения потребителей. Общей особенностью этих моделей является то, что поведение потребителей описывается только функцией спроса, зависящей либо от мгновенной интенсивности рекламных вложений фирм-участниц рынка, либо от накопленного запаса гудвилла по отношению к бренду каждой из них (интер- 
претируемого как желание потребителей купить данный товар). Основные различия между существующими моделями рекламной динамики заключаются не в механизме реакции потребителей на рекламные сообщения, а в динамике накопления гудвилла каждого из брендов.

Вышеописанные «лакуны» в исследованиях автоматически ставят перед нами задачу построения «синтетической» модели, аналогичной классической микроэкономической модели рыночного равновесия, однако с учетом того, что поведение потребителя изменяется под воздействием рекламы, а производители рассматривают рекламное воздействие как часть своей стратегии - наряду с характеристиками товара. Динамика поведения потребителей при этом может быть основана на модели адаптивного поведения, где в качестве состояния потребителей выступает выбранный ими в момент времени $t$ товар, а вероятность перехода в новое состояние (т.е. выбора нового товара вместо привычного) зависит не только от полезности текущего товара, но и от интенсивности и характера рекламы, осуществляемой каждой из фирм. Все потребители при этом разделены на несколько типов (т.е. рынок сегментирован), каждый их типов обладает собственной функцией полезности от товара, а также особенностями реакции на рекламное воздействие.

Рекламные стратегии фирм в предлагаемой модели также являются многокомпонентными. Предполагается, что в распоряжении производителей есть несколько каналов доставки рекламы до потребителей, каждый из которых имеет влияние на определенный сегмент рынка. Примером может служить рынок, на котором есть несколько телеканалов и интернет-сайты, каждый их которых сориентирован на потребителей определенной социально-демографической группы («мужчины 25-50», «женщины 35-65», и т.д.). Таким образом, компонента стратегии фирм, связанная с рекламой, описывается интенсивностью затрат на каждый их каналов доставки рекламы, а также из типа рекламы, «направляемого» в каждый из них - убеждающая, информирующая, формирующая престижность потребления, а также их всевозможные комбинации.

Еще один аспект, мало освещенный в существующих работах, связан с неполнотой производственных цепочек на рынках с рекламой. Традиционно рассматриваемые производственные цепочки на таких рынках - маркетинговые каналы - включают в себя производителей и продавцов-ритейлеров. Однако в реальности на рекламном рынке существуют полноценные игроки лица, принимающие решения - связанные с каналами доставки информации. Иными словами, телеканалы, интернет-порталы и радиостанции также являются участниками рынка, которые фактически «продают» свою аудиторию производителям. При моделировании их рыночного поведения возникает задача соединения моделей рынков классических товаров с моделями медиарынков, где реклама является уже не методом воздействия на спрос потребителей, а полноценным товаром.

Работа Бергмана и Бонатти (2011) в одном из заключительных параграфов вводит в рассмотрение модель, в которой присутствуют два канала доставки рекламы, являющиеся независимыми игроками. Они обладают стабильными распределениями аудитории, их стратегией является выбор цен на рекламу, а прибыль формируется на основе заключаемых с производителями рекламных контрактов. Кроме того, ряд работ (например, Athey et al, 2013; Ambrus et al, 
2016) посвящен построению моделей дуополии на медиарынке. В подобных моделях существуют два средства массовой коммуникации (телеканала, журнала и т.п.), принадлежащие двум фирмам-игрокам на рынке; помимо фирм, существует континуум потребителей медиа (телезрителей, читателей) и континуум бесконечно малых рекламодателей (оба множества имеют единичную массу). При этом фирмы-игроки медиарынка получают выплаты от рекламодателей в зависимости от того, какую долю аудитории среди потребителей им удастся охватить рекламными показами. Конкретный механизм взаимодействия всех участников рынка и формирования выплат медиафирмам отличаются для различных моделей. Например, в работе Эйти и соавторов (Athey et al, 2013) рассматривается двухпериодная модель, в каждом периоде потребитель контактирует ровно с одним СМИ (пусть его номер $i$ ), и видит $a_{i}$ рекламных сообщений. Рекламодатели, в свою очередь, отличаются ценностью показа рекламы для одного потребителя (а в целом их множество описывается функцией распределения на множестве допустимых значений такой ценности). Выплаты от рекламодателя компании зависят от того, какая доля потребителей хотя бы один раз увидела рекламное сообщение данного рекламодателя (общие выплаты, соответственно, равны интегралу от выплат единичного рекламодателя по всем их множеству с учетом плотности распределения).

Работа Амбруса и соавторов (Ambrus et al, 2016) посвящена схожей модели, однако в ней есть только один период взаимодействия, но потребители могут распределять свое внимание между несколькими СМИ. При этом во всех подобных моделях воздействие рекламы предполагается строго информирующим, потребитель, столкнувшийся с рекламой, лишь узнает о существовании товара, но не меняет своих предпочтений.

На применении подобного подхода (пассивные потребители и рекламодатели, игроки-медиафирмы) построен целый класс математических моделей - модели двусторонних рынков. В подобных моделях имеются две группы пользователей (аудитория-потребители и рекламодатели) и платформыпосредники, соединяющие их (медиафирмы) (Rochet, Tirole, 2003). Третичные эффекты экономического воздействия рекламы, таким образом, рассматриваются как возникающие на подобном двустороннем рынке сетевые эффекты. Стратегией медиаканалов в моделях двусторонних рынков является выбор цен на рекламу (Anderson et al, 2017, 2019), а в случае двойного финансирования (реклама + продажа контента) - еще и меню предлагаемого потребителям контента (то есть его типов и их цен для потребителя; Godes et al., 2009). Их прибыль складывается из выплат от рекламодателей контрактов (Bergemann, Bonatti, 2011; Prat, Valetti, 2018) и, если медиафирма производит и продает контент, то дополнительно и от его потребителей (Godes et al., 2009). Помимо владеющих медиа фирм существует большое количество потребителей медиа (телезрителей, читателей...) и рекламодателей. Выплаты медиафирмам от рекламодателей определяются тем, какую долю аудитории среди потребителей им удастся охватить рекламными показами. Воздействие рекламы предполагается строго информирующим, потребитель, столкнувшийся с рекламой, лишь узнает о существовании товара, но не меняет своих предпочтений. Потребитель выбирает те медиаканалы, с которыми хотел бы контактировать - ни одного, один (single-home) либо несколько (multi-home). 
Его полезность определяется, вообще говоря, как разность двух показателей собственно полезности от использования медиасервисов (например, контента (Godes, 2009) или коммуникативных возможностей социальных сетей (Prat, Valetti, 2018) и негативного воздействия, связанного с раздражением потребителя от избытка рекламы (advertising nuisance, Reisinger, 2012).

Рекламодатели в двусторонних моделях рынка представляют собой особый тип потребителей, отличных от аудитории. Задача рекламодателя заключается в том, чтобы определить оптимальный набор медиаканалов, в которых размещать свою рекламу, и объемы размещаемой в них рекламы. Критерием оптимальности является полезность, определяемая как разность вида $\psi(\mathbf{a})=E(\mathbf{a})-\Phi(\mathbf{a})$, где $\mathbf{a}-$ вектор, составленный из объемов рекламы в каждом из существующих медиа, $E(\cdot)$ - влияние такого рекламного воздействия на ожидаемую прибыль рекламодателя, а $\Phi(\cdot)$ - затраты на размещение рекламы в указанных объемах.

Основным вопросом, на который отвечают двусторонние модели медиарынков, является вопрос ценообразования на размещение рекламы в медиа и на контент. Ответ на него, естественно, существенно зависит от структуры медиарынка. Например, если существует всего одна медиафирма, то она склонна занижать цены на контент (по сравнению с немедийным монополистом, работающим на одностороннем рынке), так как это стимулирует спрос на контент и позволяет повысить цены на размещение рекламы. В то же время, для контента справедлива контринтуитивная особенность: появление на медиарынке второй фирмы может не снизить, а повысить цену на контент (Godes, 2009). В то же время, монопольная цена за размещение рекламы соответствует ожидаемым предположениям и оказывается выше олигопольной.

Очевидно, что подобные модели являются лишь начальной ступенью в моделировании реального взаимодействия СМИ как каналов доставки рекламы, производителей и потребителей, и не лишены определенной доли условности. Так, например, вопросы вызывает континуальность и одномерность распределения потенциальных рекламодателей - реальные рынки товаров с наибольшими тратами на рекламу, как правило, представляют собой олигополию, возможно, обладающую конкурентным окружением. Кроме того, весьма перспективным направлением дальнейшего развития моделей медиарынка является увеличение количества игроков со стороны СМИ - при этом их структура может быть сколь угодно сложной. Например, совокупность медиафирм может представлять собой несколько крупных медиахолдингов, каждый из которых объединяет под единым «зонтиком» большое количество изданий, телеканалов, интернет-СМИ. Поиск оптимального распределения рекламных контрактов и аудитории потребителей между отдельными подразделениями холдинга с целью максимизации совокупной прибыли каждого холдинга также является нерешенной и очень актуальной задачей. Наконец, в более обобщенной модели медиарынка медиахолдинги могут не только выступать отдельными игроками, но и пользоваться услугами медиаселлеров, представляющих их интересы и за определенную комиссию максимизирующим их прибыль (аналог картелей).

Наконец, наиболее глобальной задачей в рамках исследования экономики рекламы является построение и исследование единой трехсторонней 
модели, связывающей медиарынок, на котором «товаром» являются контакты аудитории (потребителей) с рекламными сообщениями о продукции каждого производителя, и модели рынка этой продукции. В такой единой модели одни и те же потребители выступают одновременно в двух «ролях» - потребителей товара, получающих полезность от его потребления, и потребителей медиаконтента (Вартанов, 2020). При этом потребление медиаконтента само по себе приносит им полезность (например, просмотр интересного кино по телевизору или чтение интересной статьи в журнале), одновременно с этим позволяя производителям товаров оказывать на них рекламное воздействие. Фирмы-владельцы СМИ представляют собой третий тип участников рынка, отличающийся как от производителей, так и потребителей - они одновременно генерируют контент, продавая его потребителям, и доводят до них рекламу производителей, «продавая» им свою аудиторию. Именно в возникновении отдельного медиарынка, нераздельно связанного в единый метарынок с рынками всех товаров, нуждающихся в рекламе, и заключается «третичный» эффект рекламного воздействия на рынок - эффект более высокого, чем первичный (изменение поведения потребителей) и вторичный (изменение структуры товарного рынка без изменения ролей его участников), уровня.

\section{Заключение}

В настоящей работе были кратко проанализированы и классифицированы существующие в настоящее время взгляды и подходы к описанию феномена рекламы и эффектов от его воздействия на различных уровнях экономики. Все подобные эффекты были разделены по трем уровням - в зависимости от того, на агентов какого уровня влияет появление рекламы на рынке. К первичным были отнесены все те эффекты, которые реклама оказывает на поведение конечных потребителей: эффекты рекламной коммуникации, эффекты убеждения и информирования, а также поведенческие эффекты (фрейминг, отвлечение и т.д.), используемые при проведении промоакций и проектировании множества выбора. Вторичные эффекты связаны с изменением поведения производителей и фирм, в руках которых оказываются рекламные инструменты. К третичным эффектам относятся глобальные изменения свойств рыночного равновесия и рыночной структуры в связи с появлением на рынке рекламы - от изменения общественного благосостояния до появления полноценного рынка рекламы и медиа и сетевых эффектов, возникающих на них. Настоящая работа представляет собой первую из нескольких статей и описывает общую классификацию эффектов рекламы, последующие публикации посвящены подробному обзору литературы, исследующей эффекты каждого из уровней.

\section{Список источников / References}

1. Ambrus A., Calvano E., Reisinger M. (2016). Either or both competition: A" twosided" theory of advertising with overlapping viewerships // American Economic Journal: Microeconomics, Vol. 8(3), 189-222.

2. Anderson S. P., Foros Ø., Kind H. J. (2017). Competition for advertisers and for viewers in media markets // The Economic Journal. Vol. 128 (608), 34-54. 
3. Anderson S. P., Foros Ø., Kind H. J. (2019). The importance of consumer multihoming (joint purchases) for market performance: Mergers and entry in media markets // Journal of Economics \& Management Strategy. Vol. 28 (1), 125-137.

4. Athey S., Calvano E., Gans J. (2013) The impact of the internet on advertising markets for news media. - National Bureau of Economic Research. - №. w19419.

5. Bergemann D., Bonatti A. (2011) Targeting in advertising markets: implications for offline versus online media // The RAND Journal of Economics. Vol. 42 (3), 417-443.

6. Chamberlin E. (1933) The theory of monopolistic competition: Awarded the David A. Wells prize for the year 1927-28, and published from the income of the David A. Wells fund. - Harvard University Press.

7. Dorfman R., Steiner P. O. (1954) Optimal advertising and optimal quality // The American Economic Review. 1954. Vol. 44 (5). 826-836.

8. Godes D., Ofek E., Sarvary M. (2009). Content vs. advertising: The impact of competition on media firm strategy // Marketing Science. Vol. 28 (1), 20-35.

9. Huang, J., Leng, M., \& Liang, L. (2012). Recent developments in dynamic advertising research // European Journal of Operational Research, 220, 591-609.

10. Jørgensen S., Zaccour G. (2014) A survey of game-theoretic models of cooperative advertising // European Journal of Operational Research. - T. 237. - №. 1. - C. 1-14.

11. Nerlove M., Arrow K. J. (1962) Optimal advertising policy under dynamic conditions // Economica, 29(114):129.142.

12. Prasad A., Sethi S. P. (2009) Integrated marketing communication in markets with uncertainty and competition // Automatica, 45(3),601-610.

13. Prat, A. and Valletti, T. M. (2018). Attention oligopoly. // Mimeo.

14. Ray, M. (1973). Marketing communication and the hierarchy of effects. Stanford University Graduate School of Business. Research Paper No. 180.

15. Reisinger M. (2012). Platform competition for advertisers and users in media markets // International Journal of Industrial Organization. Vol. 30 (2), 243-252.

16. Rochet J. C., Tirole J. (2003). Platform competition in two-sided markets // Journal of the European economic association. Vol. 1 (4), 990-1029.

17. Vidale L., Wolfe H. B. (1957) An operations-research study of sales response to advertising // Operations Research. Vol. 5(3):370.381, 1957.

18. W. S. Comanor and T. A. Wilson. (1974) Advertising and Market Power. Harvard University Press, Cambridge, MA.

19. Вартанов С. А. (2020) Математическое моделирование трехстороннего рынка: медиа, производство и потребители // Известия Дальневосточного федерального университета. Экономика и управление. - № 1. - С. 22-37.

20. Назайкин, А.Н. (2014). Как оценить эффективность рекламы. Москва: Солонпресс.

\section{Сведения об авторе / About author}

Вартанов Сергей Александрович, кандидат физико-математических наук, доцент кафедры эконометрики и математических методов экономики Московской школы экономики, Московский государственный университет имени М.В. Ломоносова. 119234 Россия, Москва, Ленинские Горы, д.1, стр. 61

E-mail:sergvart@gmail.com

Sergey A. Vartanov, Candidate of Physical and Mathematical Sciences, Associate Professor at the Chair of Econometrics and Mathematical Methods in Economics, Moscow School of Economics, Lomonosov Moscow State University. Bldg. 61, 1 Lenin Hills, Moscow, Russia 119234.

E-mail:sergvart@gmail.com 